\title{
BIOMIMETIC STRATEGIES IN ORGANIC SYNTHESIS. TERPENES
}

\author{
Veaceslav Kulciţki \\ Institutul de Chimie al AŞ a RM, str. Academiei, 3, MD-2028, Chişinău, Republica Moldova \\ E-mail:kulcitki@yahoo.com
}

\begin{abstract}
The current paper represents an outline of the selected contributions to the biomimetic procedures and approaches for the synthesis of terpenes with complex structure and diverse functionalisation pattern. These include homologation strategies, cyclisations, rearrangements, as well as biomimetic remote functionalisations. Diverse groups of terpenes have been made accessible by the reported procedures: seco-eudesmanes, sacculatanes, cheilanthanes, scalaranes, rearranged labdanes and austrodoranes. Most of the presented synthetic methods represent relevant preparative value, due to the simplicity and reduced number of steps.
\end{abstract}

Keywords: terpenoids, biomimetic synthesis, cyclisation, rearrangement, oligomerisation, functionalisation.

\section{Introduction}

Organic chemistry represents one of the basic areas of modern science. When we make an outline of the reasons which define the special place of this area of human knowledge, its two general aspects come into mind: cognitive and creative power. Both these aspects are quite clear. First of all we strive in our endeavours to explain all the processes which surround us. Chemists see the life through the prism of chemical reactions: reactions that occur in our own bodies, reactions that occur in the invisible micro-world, reactions that permanently affect our environment and finally reactions that take place in the outer space. This cognitive function inevitably leads to our desire to create new matter, to produce materials and substances that we might consider useful for our lives. And the pivotal role of organic synthesis in this process is generally acknowledged. It is due to the practically infinite range of compounds which can be built on the basis of organic carbon chains. But what is the strategy which leads the chemists into this creative Odisea? Again, two distinct approaches are valid: we create something that we think it might be usefull and we create what we see in the nature. The second approach in fact reflects one of the basic psychological features of humans as social beings: we build our lives by mimicking our environment. For organic chemists this means identifying natural targets and their reproduction. Following the same strategy, we try to hypothesise the in vivo biochemical pathways leading to the targets and then to reproduce similar synthetic sequences in vitro. This is called a biomimetic strategy.

A careful examination of the chemicals that have firmly entered our lives will make us conclude that most of them are mimics of natural matter: from low molecular wait bio-regulators (pheromones, drugs, agrochemicals) to polymers and supramolecular aggregates (textiles, rubber and plastics). A comprehensive study of the examples illustrating this reality would certainly do not fit the range of a journal paper. The purpose of the current report is to provide just a single example of biomimetic approach in developing synthetic strategies. This is the example of terpenes - a very large and fascinating group of natural compounds.

\section{Terpenes. Structure and biosynthesis}

Terpenes represent a large family of natural products with an impressive diversity of carbon skeletons and functionalisation pattern. It is believed that this diversity is due to the latter steps of terpene biosynthesis [1]. This opinion has been confirmed by numerous biosynthetic studies which proved that common precursors of all terpenic families are only several open chain oligomers of dimethylalylpyrophosphate (DMA-OPP): geranylpyrophosphate, farnesylpyrophosphate, geranylgeranylpyrophosphate and some other superior oligomers. The biosynthetic pathways to these basic building blocks include two steps. The first one is the synthesis of DMA-OPP either by mevalonate (MP) or methylerythritolphosphate (MIP) pathways (Scheme 1), which along with its precursor isopentenylpyrophosphate (IP$\mathrm{OPP})$ represent the elementary $\mathrm{C} 5$ terpene units. 
MP:
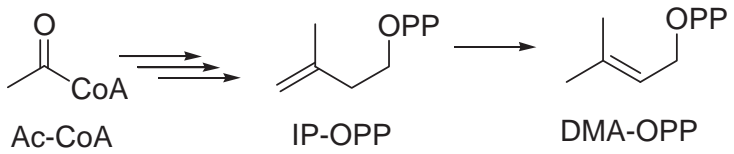

MIP:

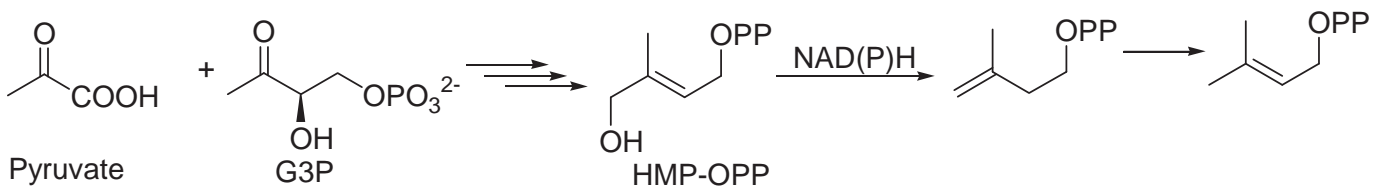

Scheme 1. Early stage terpene biosynthesis. Mevalonate (MP) and mevalonate-independent (MIP) pathways

The second step of biosynthesis is the coupling of C5 fragments leading to the corresponding open chain mono-, sesqui-, di- and polyisoprenoids. DMA-OPP and IP-OPP combine in an oligomerisation process to form C10, C15, C20 and higher aliphatic chains lake geraniol (C10), farnesol (C15), geranylgeraniol (C20) and higher polyprenols.

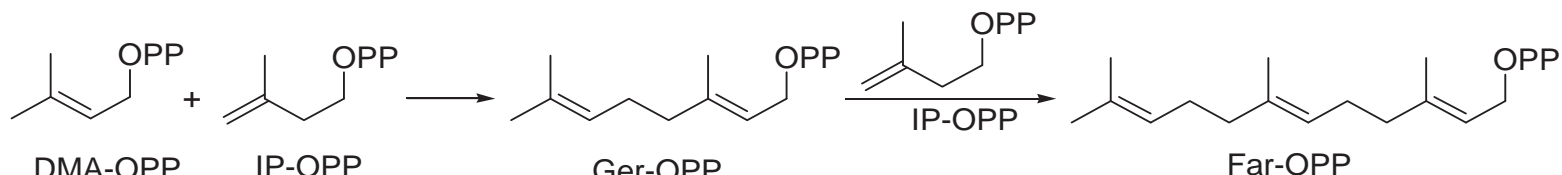

Scheme 2. Oligomerisation of isoprene units

Both these steps are similar in all producing cells and living organisms and they bring no structural diversity. On the contrary, the next two steps of terpene biosynthesis play the crucial role in the tremendous expansion of possible terpene structures. These steps are cyclisations/isomerisations and selective functionalisations/degradations mainly by oxygenated functionalities. The enzymes responsible for these transformations are terpene cyclises and oxidases. From the mechanistic point of view, the terpene cyclases represent an interesting subject, since their action is accompanied by a huge variety of other transformations, which besides cyclisations may include hydride shifts, Wagner-Meerwein and other skeleton rearrangements. Carbonium ion intermediates are the species that ensures reaction sequences leading to carbon skeleton diversity. To date, there are two basic mechanisms of terpene cyclisations.

The first mechanism is given by the ability of the double bond to act as a nucleophile and to interact in a SN2 fashion with the $\alpha$-terminus of the chain, leading to elimination of the -OPP group and formation of a new C-C bond.

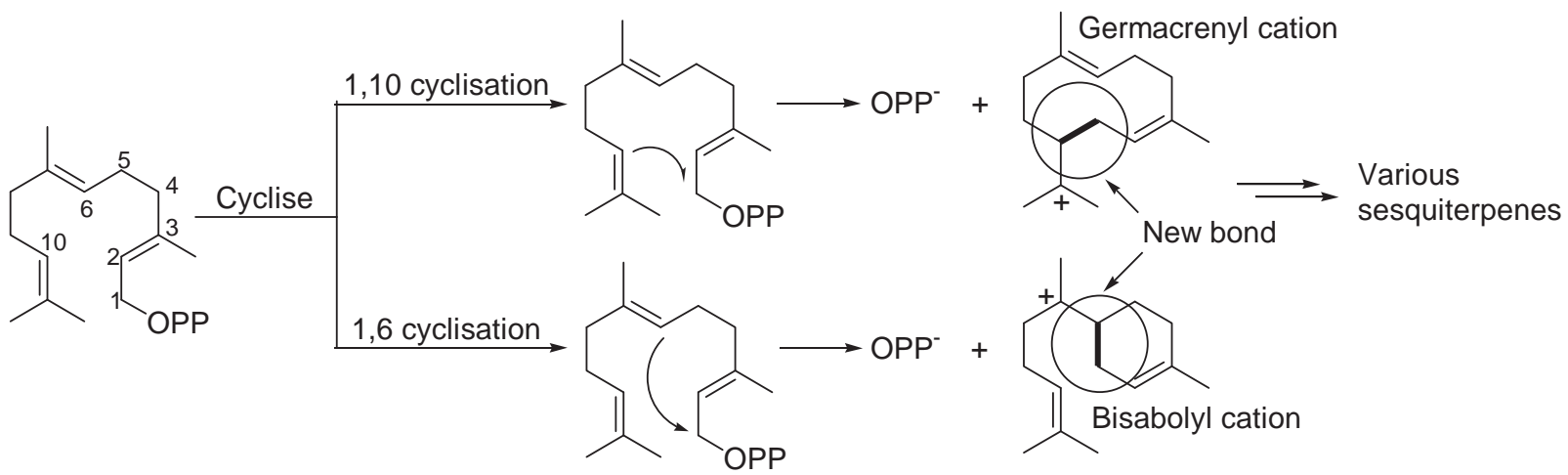

Scheme 3. Cyclisation of terpenes by a nucleophilic substitution of the-OPP group

The Scheme 3 presents two different paths for the cyclisation of farnesyl-OPP, leading either to germacrenylor bisabolyl- cations. Both these paths occur according to this mechanism, involving either of the double bonds present in the chain.

The second mechanism includes a cascade of reactions initiated by a selective protonation of the double bond, followed by a electrophilic attack of the formed carbonium ions to the other double bonds of the aliphatic chain (Scheme 4). Both mechanisms involve formation of intermediate carbonium ions, which can stabilize either by proton elimination, skeletal rearrangements or addition of other nucleophiles. 


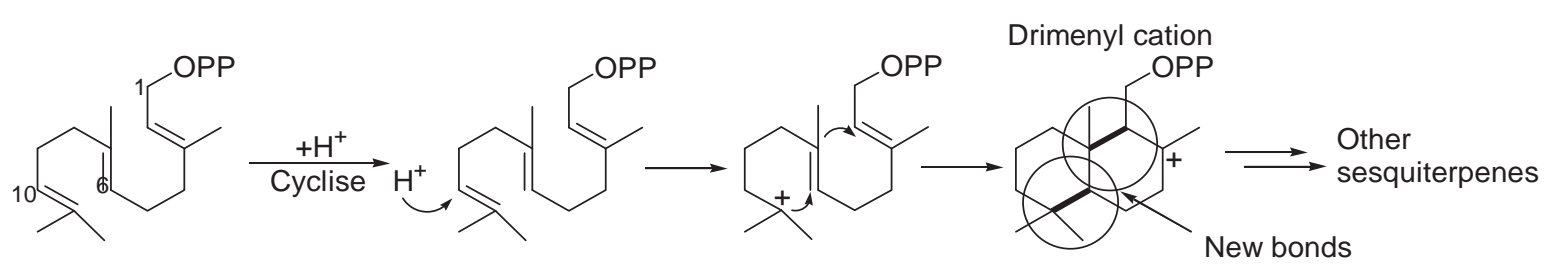

Scheme 4. Electrophilic ciclisation of terpenes by selective protonation

And the third important process which defines the structural diversity is based on the selective functionalisation of terpenes by different enzymes, which introduce additional heteroatoms, basically by an oxidative process (Scheme 5). Intercalation of cyclisations, isomerisations and functionalisations provides practically infinite opportunities for structural modification of the terpenes in living organisms.

All the enumerated biosynthetic processes have inspired organic chemists to devise synthetic methods which selectively transform the substrates exactly in the same way as enzymes do, that is to mimic the biosynthesis.

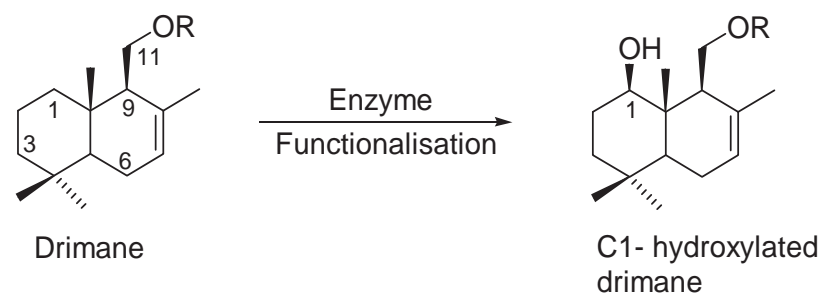

Scheme 5. Enzymatic terpene functionalisation

Surprisingly, but for some instances chemical processes have been devised long before the identification of corresponding enzymes. Baiyer-Williger oxidation of ketones and Diels-Alder cycloadditions are relevant examples. Table 1 presents a short outline of the most known processes which are successfully applied in biomimetic synthetic strategies.

Table 1

Bio- versus chemical synthesis.

\begin{tabular}{|l|l|}
\hline \multicolumn{1}{|c|}{ Enzyme } & \multicolumn{1}{c|}{ Biomimetic process } \\
\hline Preniltransferaze & Oligomerization \\
Cyclaze & Cyclisation \\
Citocrom P450 & Oxidation \\
Diels-Alderaze & Cycloaddition \\
Baeyer-Villigeraze & Baeyer-Villiger oxidation \\
Oxidoreductaze & Reduction \\
\hline
\end{tabular}

Examples of synthetic schemes devised according to these strategies are numerous. The following selection is provided to illustrate the most relevant ones, when used for the synthesis of complex terpenic compounds in a selective manner.

\section{Oligomerisation}

The need to do terpenes oligomerisation in vitro is dictated by the fact that terpene raw materials are quite limited to lower terpenes. Economically significant and renewable resources for terpene isolation are the wastes of wood processing industry first. The major part of these wastes are monoterpenes. The use of other important higher natural terpenoids like sclareol or manool is also connected to the need of oligomerisation, that's why elaboration of synthetic processes aimed to linkage of terpenic units has represented a priority of the research groups working in this field. The basic strategy relies on a biomimetic modular approach of connecting a $\alpha$-functionalized building block to a $\alpha, \omega$-bifunctional fragment (Scheme 6). Due to practical reasons, the $\alpha$-functionalized fragment bears more complexity (cyclic structures, chirality, functional groups), and the $\alpha, \omega$-bifunctional fragment is a simple C5 or C10 unit. 


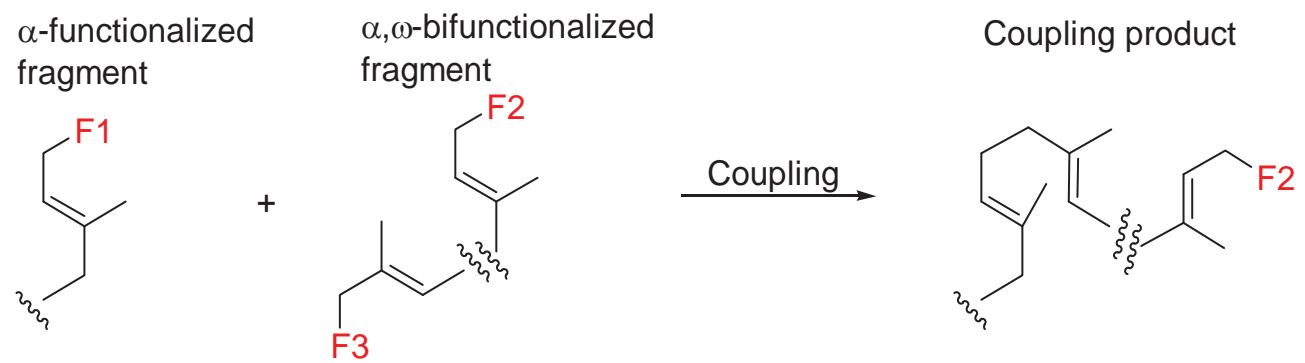

F1-F3: functional groups

Scheme 5. Strategy of terpene oligomerisation by synthesis

The activating functional groups F1 and F3 are selected in order to provide a couple of donor-acceptor synthons, which are able to efficiently combine and form the new $\mathrm{C}-\mathrm{C}$ bond. Lithiated sulfones are used as synthetic equivalents for donor synthons and halogenides or carbonyl compounds for acceptor ones.

This strategy was successfully reported by different research groups [2] due to its several advantages. First of all the introduction of required sulfone and halogenide functional groups can be easily performed by routine techniques. The coupling yields vary from good to excellent and the products incorporate the sulfone group which can be handled in a flexible manner: either substituted for a hydrogen atom or for other functional groups [3]. In a couple of recent examples this strategy was successfully used in our laboratory [4]. The farnesylchloride 1 was coupled with the lithiated sulfone 2 derived from geraniol to provide the all-trans sesterterpene 3 (Scheme 6). Using the optically active drimenylbromide 4 as coupling partner for $\mathbf{2}$, generated a coupling product $\mathbf{5}$, incorporating two elements of complexity: bicyclic fragment and chirality.

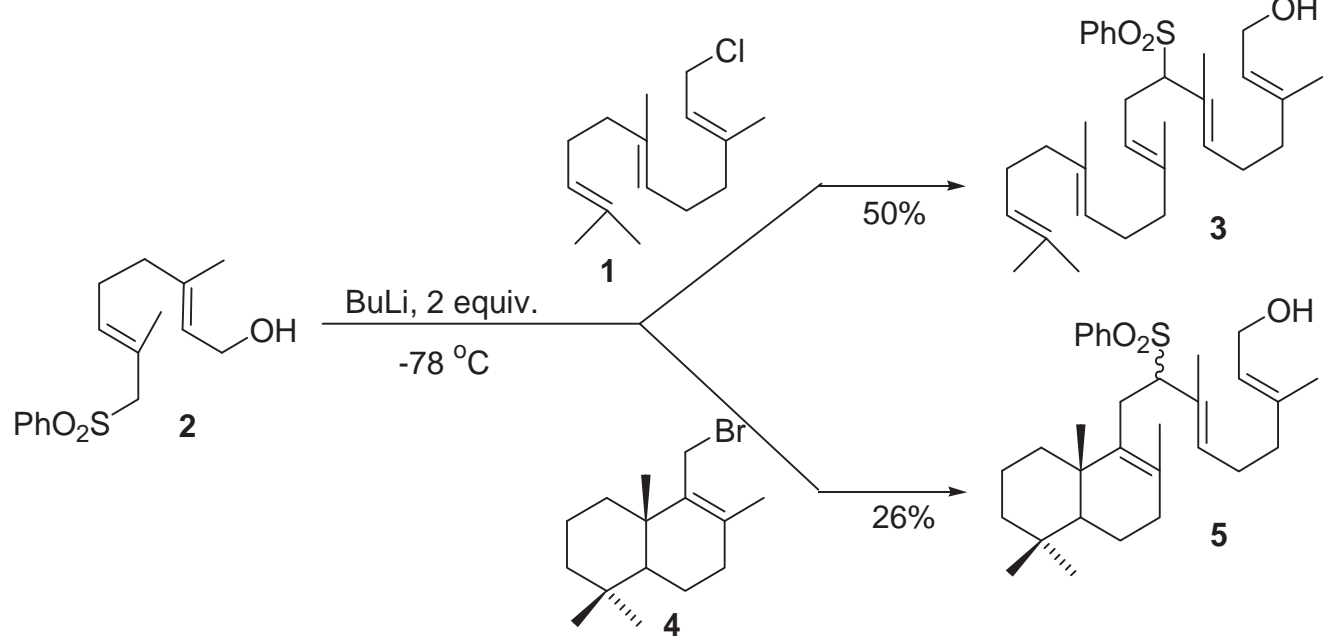

Scheme 6

This homologation method also showed excellent regio-flexibility. The reported synthesis of natural product trans16-hydroxygeranylgeraniol $\mathbf{6}$ included a similar coupling of $\mathbf{7}$ and $\mathbf{8}$, with the sulfone group placed on the $\alpha$-terminus and the chlorine on the $\omega$-terminus [5]. The yield of $\mathbf{9}$ was excellent (Scheme 7).

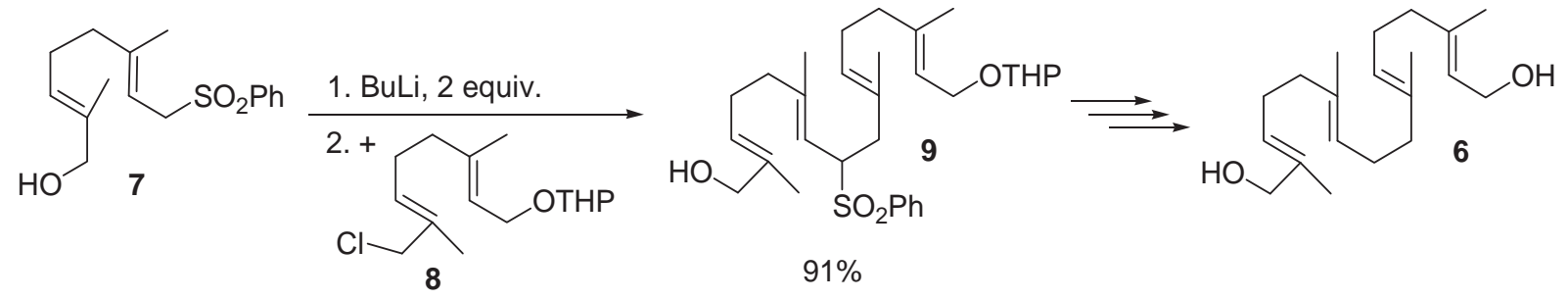

Scheme 7 


\section{Cyclisations}

Terpenoid cyclisations represent the classical example of biomimetic approach in the synthesis. The major part of the elaborated reactions is based on the mechanism including carbonium ion generation via selective protonation (second biosynthetic pathway, Scheme 4). Different cyclisation agents have been reported, most of them possesses strong acidic properties. The major challenges connected to this strategy relates to the need of controlling reaction selectivity, since the number of reaction pathways, and the range of possible products increases dramatically along with the number of isoprene units of the molecule. Therefore, cyclisation methods evolved to the use of lower reaction temperatures and stronger acid initiators. It is understandable that lower temperature influences the conformational flexibility of the molecule, in an attempt to mimic the action of the enzyme, which practically locks the substrate in one single conformation, making the cyclisation process totally specific. Solid acids like zeolites and acidic resines have been also reported to contribute to this problem solution. Their advantage consists in reaction conditions close to ambiental but unfortunately their selection is mostly empirical, they are very substrate-specific, thus limiting the process versatility. More practical from this point of view turned out to be superacids [6,7], which can initiate cyclisation sequences at temperatures as low as $-78^{\circ} \mathrm{C}$ or even lower. Consequently, the process selectivity is beneficially influenced. A significant advancement in this field represents our finding that additional functional groups placed at certain positions of aliphatic terpenic chain, can influence the reaction course, allowing either selective initiation or termination of the cascade. The pioneering work relating in this directions deals with the superacidic cyclisation of $\alpha, \omega$-bifunctionalized sesquiterpenic substrates [8]. The presence in the substrate $\mathbf{1 0}$ of the acetoxy-group at the $\omega$-terminus hindered the initiation of the cyclisation sequence from the extreme isoprene unit and allowed a selective protonation of the middle double bond, leaving in the resulted product $\mathbf{1 1}$ a pendant prenyl-group attached to the monocyclic backbone (secoeudesmanic structure, Scheme 8).<smiles>CC(=O)OCC1=CCCC1=CCCC(C)=CCOCc1ccccc1</smiles>

10

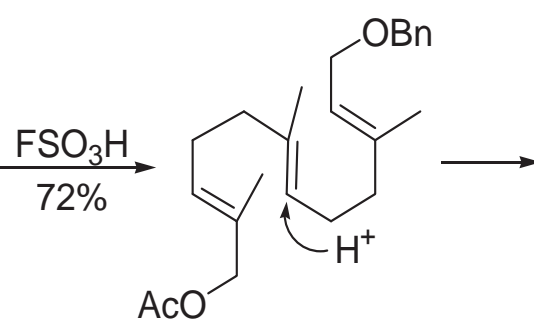

Scheme 8

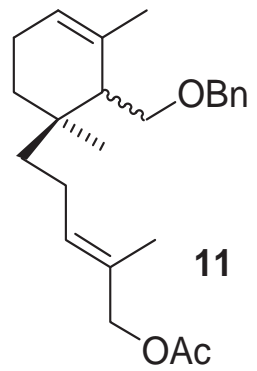

This reaction mechanism was further exploited for the biomimetic synthesis of sacculatane-like diterpenoids [9]. The $\alpha, \omega$-diterpenic open chain substrate 12 was prepared from geranylgeraniol, basing on a selective Van Tamelen epoxidation, followed by a degradation-olefination sequence. Superacidic treatment of $\mathbf{1 2}$ at low temperature allowed isolation of sacculatane-like bicyclic compounds as major reaction products (Scheme 9).

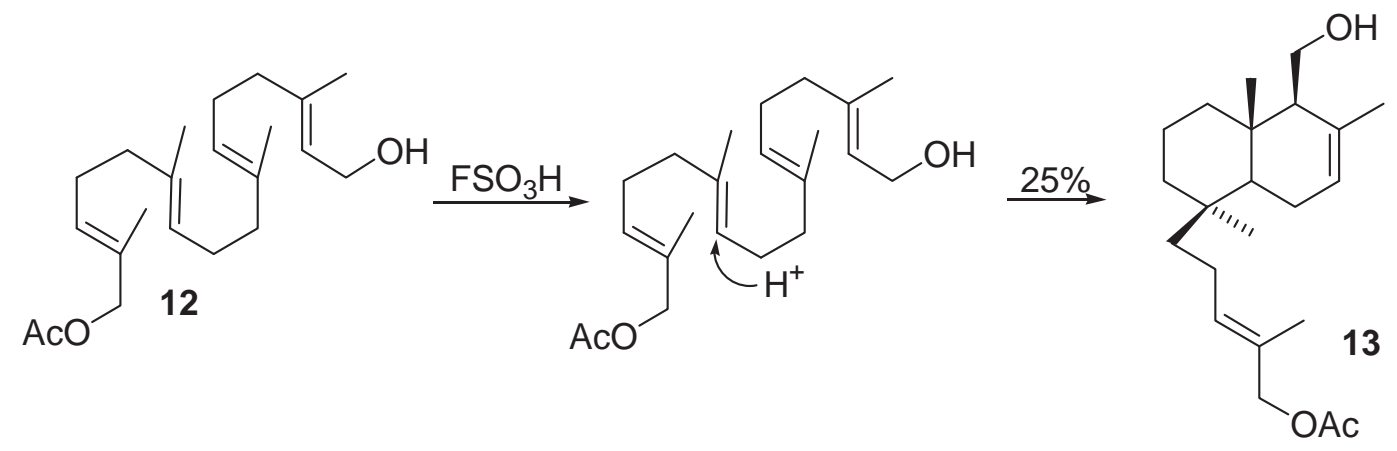

Scheme 9

Despite of the relatively low yield of the target $\mathbf{1 3}$, the value of this example is given by the unique reaction mechanism, which proves the principle of functional group involvement into directing cyclisation selectivity. It is noteworthy mentioning that a substrate analogue to 12 , but without a functional group at $\omega$-end, provides under similar conditions a totally cyclized tricyclic compound [6]. 
A totally opposite cyclisation pattern has been revealed on the investigation of terpenic substrates containing double bond with alternating configuration (trans-vs. cis-). The impetus for these investigations was provided by a remarkable class of natural isoprenoids - polyprenols. These compounds are higher oligomers, containing from 5 to 11 or even more isoprene units. They are usually found in plants and are potentially regarded as precursors of condensed polycyclic substances found in fossil sediments. In order to check the feasibility of this idea, we have initiated a program on superacidic isomerisation of selected polyprenols. The most representative di-trans-poly-cys- substrates have been chosen.

As it was expected, all the subsrates, independently on the chain length have shown reactivity on superacidic treatment. But the conclusions on the reaction mechanism could have been drawn only for a lowest $\mathrm{C}-25$ representative 14 [10]. Selection of the esteric group as $\alpha$-terminus functionality was due to its relative stability to superacidic treatment and also to the fact that long chain polyprenols showed a pronounced tendency to sediment at low temperature in the reaction solvents. The synthesis of $\mathbf{1 4}$ was performed from commercial geranyllinalool 15, making use of a Carroll rearrangement followed by separation of the cis-ketone $\mathbf{1 6}$ and its standard olefination (Scheme 10). This two-step homologation procedure was preferred, since the Carroll rearrangement provided a significant ratio of the required $\mathbf{1 6}$.<smiles>C/C=C/C(C)(O)CC/C=C1\CC/C=C(/C)CCC1</smiles>

15

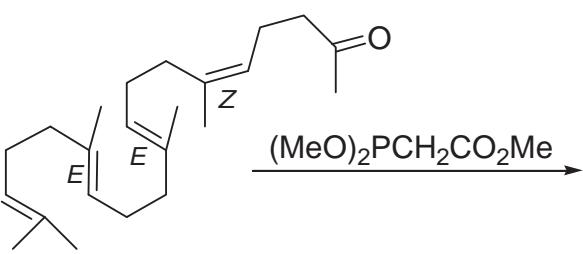

16

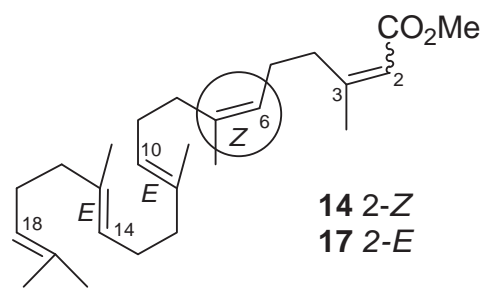

Di-trans-poly-cyspolyprenol-like substrate

Scheme 10. Synthesis of polyprenol-like compound with the $6-Z$ double bond

The cyclisation of both substrates 14 and $\mathbf{1 7}$ occurred smoothly on treatment with fluorosulfonic acid (Scheme 11). Predominant reaction products in both cases were tricyclic compounds $\mathbf{1 8}$ and 19, having the $\alpha$-prenyl unit pendant. These compounds belong to the cheilanthane family and their biomimetical synthesis turned out to be a very efficient preparative tool. In fact, the use of substrate $\mathbf{1 4}$ as starting material shows clearly that cyclisation of an open chain polyprenol-like substrate can be suspended to a tricyclic compound, due to the presence of an internal cis-double bond. This functionality plays a crucial role in the conformational behavior of the substrate, braking-up the cyclisation cascade via a complex conformational-steric effect.

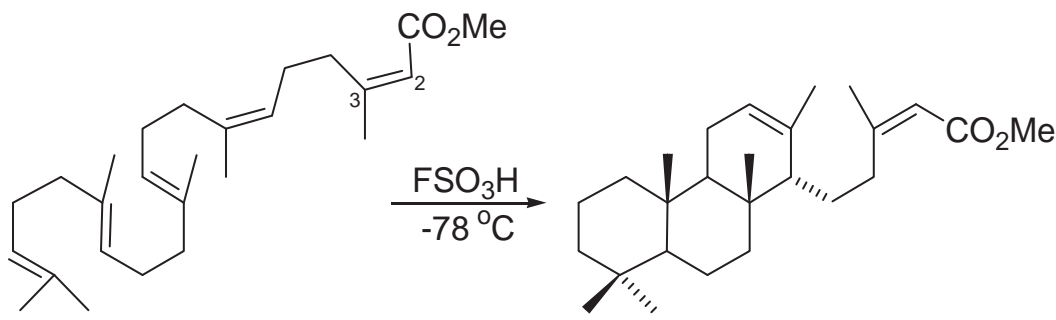

14

$1839 \%$<smiles>CCC=C(C)CCC=C(C)CCC=C(C)CCC=C(C)C</smiles>

17

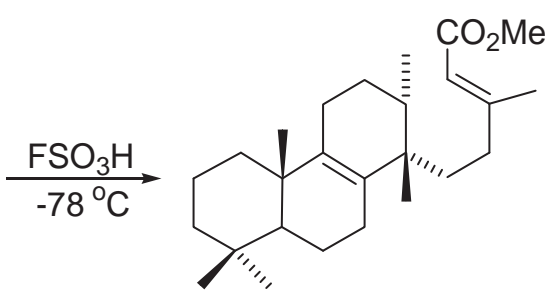

$1963 \%$

Scheme 11. Superacidic cyclisation of polyprenol-like substrates. Biomimetic synthesis of cheilanthanes

This finding led to the idea of using more available bicyclic starting materials for preparation of cheilanthanes in optically active form [11]. Labdanic diterpenoid sclareol 20 was the candidate of choice. It represents a relatively cheap compound, produced commercially as a by-product of Salvia sclarea essential oil processing. 


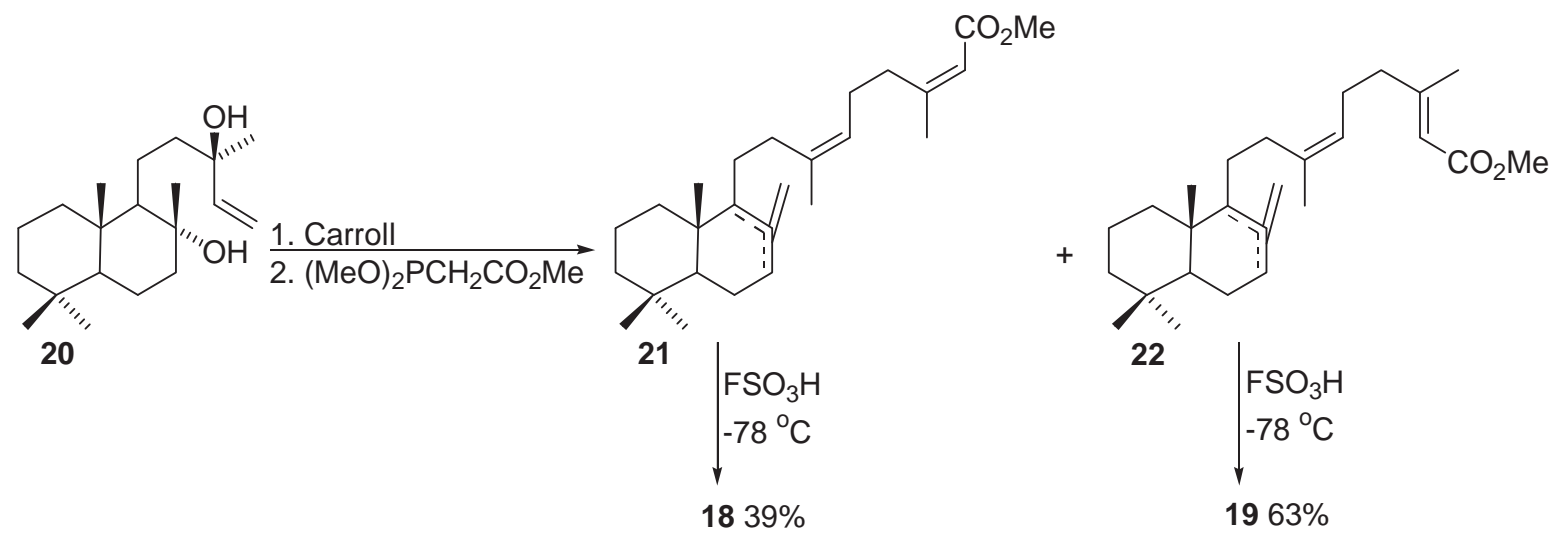

Scheme 12. Biomimetic synthesis of optically active cheilanthanes

Transformation of $\mathbf{2 0}$ into the cyclisation substrates $\mathbf{2 1}$ and 22 followed the same synthetic sequence shown in Scheme 10: Carroll rearrangement, followed by Horner-Wadsworth olefination. Cyclisation of 21 and 22 proceeded smoothly, to provide cheilanthanes $\mathbf{1 8}$ and $\mathbf{1 9}$ in optically active form (Scheme 12). This approach represents a complementary addition to the alternative syntheses of cheilanthanes $[12,13]$ and has a great synthetic value due to its simplicity and reduced number of steps. Besides, it is also a reasonable method for the synthesis of scalaranic compounds, which are important by-products $[14,15]$ formed in substantial amounts along with cheilanthanes $\mathbf{1 8}$ and 19. Our finding, that formation of scalaranes is not influenced by the $E-Z$ configuration of the internal double bond has allowed elaboration of an efficient method for the synthesis of scalaranes and cheilanthanes in an integrated process. Its value is given by the possibility of separation the scalaranic compounds from cheilanthanic without chromatography. The flowchart of this procedure is represented in Scheme 13. After batch cyclisation of $\mathbf{2 1}$ and 22, hydrolysis of the reaction products lead to scalaranic esters which do not hydrolyse under the reaction conditions, and cheilanthanic acids. Separation of the acidic and neutral part gives intact scalaranic esters in neutral fraction, while cheilanthanic acids can be recovered from the acidic fraction.

$$
\text { 20 } \underset{\text { 1. Carroll }}{\text { 2. }(\mathrm{MeO})_{2} \mathrm{PCH}_{2} \mathrm{CO}_{2} \mathrm{Me}} 21+22 \underset{-78}{\stackrel{\mathrm{FSO}_{3} \mathrm{H}}{\longrightarrow}}
$$

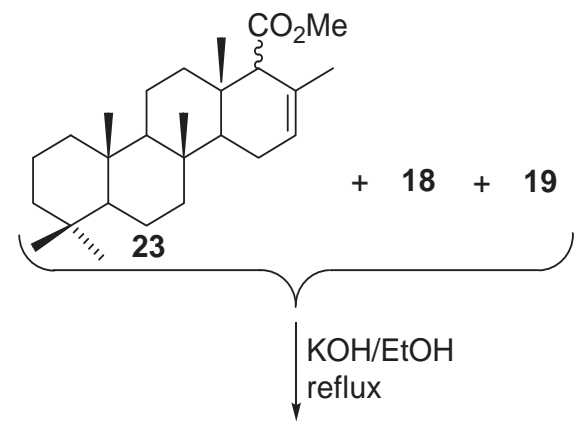

Unreacted $23+$ Acidic part (cheilanthanes)

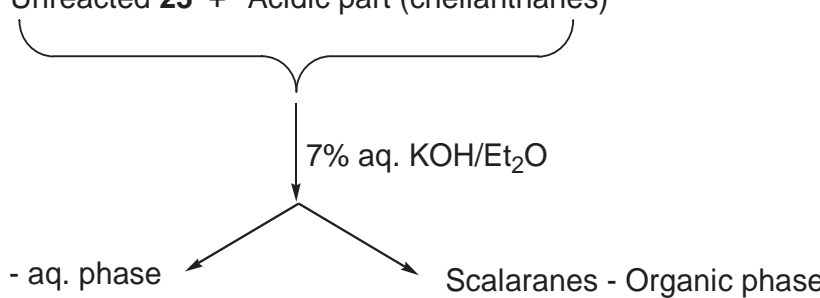

Scheme 13. A preparative procedure for the synthesis of scalaranes and cheilanthanes in optically active form

\section{Rearrangements}

A whole range of terpenic compounds are biosynthetically formed by rearrangement reactions. These can include ring contractions, ring expansions and even functional group migrations. A good source of such rearranged terpenes are marine organisms, which provide constantly inspiration to synthetic organic chemists, due to the enormous diversity of unusual structures reported from this relevant source of natural compounds. Spongiane diterpenoids derive from sponges and besides their normal tetracyclic framework $\mathbf{2 4}$, a lot of these derivatives resemble degraded skeletons. 
Norrisolide 25, a complex Golgi fragmentation agent isolated from the nudibranch Chromodoris norrisi, was postulated to derive biosynthetically from spongianes, by a complex degradation-rearrangement sequence [16]. The simpler norsesquiterpenic austrodoral 26, isolated from the dorid nudibranch Austrodoris kerguelenensis was also suggested to arise biosynthetically from the drimane skeleton 27 by a ring-contraction process [17].<smiles>C=CC(=O)[C@H]1CCC2C(C)(C)CCC[C@]21C</smiles>

25<smiles>CC1(C)CCC[C@]2(C)C3CCC4COCC4[C@@H]3CC[C@@H]12</smiles>

24<smiles>C=C[C@]1(C)CCC2[C@]1(C)CCC[C@@]2(C)C=O</smiles>

26<smiles>[R]CC1C(C)CCC2C(C)(C)CCC[C@]12C</smiles>

27

Basing on this hypothesis, a biomimetic procedure for the synthesis of $\mathbf{2 6}$ was devised, starting from drimanic or hoomodrimanic starting materials $[18,19]$.

The sequence of key transformations leading to 26 is represented in Scheme 14. The key reaction was a biomimetic ring contraction of the suitable constructed epoxide under the action of a Lewis acid to a ring contracted, perhydrindanic ketone 30.<smiles>CC(=O)OC[C@H]1[C@@](C)(O)CC[C@@H]2C(C)(C)CCC[C@@]12C</smiles>

28<smiles>CC(=O)OC[C@]1(C)[C@@H](C)CC[C@@H]2C(C)(C)CCC[C@]21C</smiles>

29<smiles>CC(=O)OCC(=O)[C@@]1(C)CCC2C(C)(C)CCC[C@]21C</smiles>
$\underset{\text { 1. } \mathrm{LAH}}{\stackrel{\mathrm{NaIO}}{4}}$ 30<smiles>CC1(C)CCC[C@@]2(C)C1CC[C@@]2(C)C=O</smiles>

26

Scheme 14. Synthesis of austrodoral 26 from drimanes by a biomimetic ring contraction strategy

Elimination of the additional carbon atoms was performed by a standard periodate cleavage of the corresponding diols.<smiles>CC(=O)OCC[C@]1(C)[C@@H](C)CCC2C(C)(C)CCC[C@]21C</smiles>

33<smiles>CC(=O)OCCC(=O)[C@@]1(C)CCC2C(C)(C)CCC[C@]21C</smiles>

34<smiles>C=C1CCC2=C(CCCC2(C)C)[C@@]1(C)CCOC(C)=O</smiles>

35

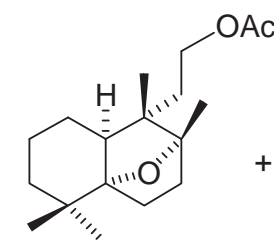

36<smiles>CC(=O)OCC[C@@]1(C)C2=C(CC[C@]1(C)O)C(C)(C)CCC2</smiles>

37<smiles>CC1(C)CCC[C@@]2(C)[C@H]1CC[C@]2(C)C(=O)O</smiles>

32

Scheme 15. Rearrangements of homodrimanes

Using the homodrimanic substrate $\mathbf{3 3}$ as starting material, provided the related austrodoric acid $\mathbf{3 2}$, but unlike drimanic epoxide 29, the homodrimane 33 interacted with different acidic inducers in a less selective manner. The perhydrindane 34 represented the basic product, but other compounds of deeper skeletal rearrangement have also been identified in the reaction mixture (Scheme 15). It included a cascade of methyl migration-hydride shifts-proton eliminations-heterocyclisation leading to halimanic bicyclic core $[20,21]$. Variation of the reaction conditions, including reaction temperature and nature of acid inducer allowed a moderate selectivity control towards either ring contraction or methyl migration paths. This example incorporates two different biogenetic pathways towards compounds of both perhydrindanic and halimanic structure. The striking diference in the reactivity of drimanic 29 and homodrimanic 33 substrates is explained by the involvement of the lateral chain in the intermediate carbonium ions stabilization. 


\section{Selective functionalisation}

The last chemical phenomenon under discussion is directly connected to the functional properties of terpenes, due to the heteroatoms incorporated into the carbon skeleton formed by oligomerisation-cyclisation-isomerisation sequences. Introduction of functional groups plays the crucial role in the interaction of terpenes with biological matrix leading to certain activities of the molecules. Oxigenated functionalities are the most frequently met as terpene "decoration", but nitrogen and halogens play an important role too.

Biomimetic approach can be implemented by two different ways for terpene functionalisation. The first mode includes selective functionalisation of the ready-made terpenic skeleton. The advantagies of this post-cyclisation process are connected first of all to the fact that cyclic terpenic molecules contain less reactive double bonds, and functionalisation can be more selective, at the expense of lower reactivity. Besides, cyclic molecules possess a relatively rigid molecular conformation, which can enhance steric control over functionalisation process.

The second functionalisation strategy is based on the fact that we do not always know exactly the sequence of biosynthetical transformations and it is possible to hypothesize that functionalisation can occur before cyclisation/ rearrangement in biosynthesis. In fact, additional functional groups in the substrate would inevitable control the nature and selectivity of both enzymatic and chemical transformations, including cyclisations/rearrangements. Therefore, it can be used as a tool to control the synthetic process too. On the other hand, additional functional groups in the terpenic molecule influence inevitably its steric and electronic features and shall be carefully handled. From the practical point of view, introduction of additional functionalities in the non-cyclized or partially cyclized molecule is more convenient, since the isoprene residues double bonds can be efficiently exploited for functionalisation purposes. The disadvantages of this strategy are connected to the difficulties of prediction the effect of the functional groups on the following reactivity.

Both these functionalisation pathways have been exploited in our works and the results are encouraging. First of all it was investigated the so-called remote functionalisation of a scalaranic tetracyclic compound $\mathbf{3 8}$, having the carbon skeleton already established in place by a oligomerisation-cyclisation sequence [22]. Our goal was to set up an oxygenated functional group in the B-cycle of the tetracyclic system of 38. Due to the fact, that this cycle contains only C-C and $\mathrm{C}-\mathrm{H}$ bonds which can hardly be selectively transformed, the only feasible way to achieve selective functionalisation was considered the so called radical-relay halogenation (RRH) [23]. The characteristic feature of this reaction is the generation of a free radical in the molecule of the substrate, that has to contain a suitable functional group playing the role of a 'relay' for the free radical formation (radical "handle", Scheme 16), followed by the functionalization of nearby disposed $\mathrm{C}-\mathrm{H}$ bond by this radical. The position of functionalization is governed both by steric and structural factors (only tertiary protons are prone to be involved into this process).
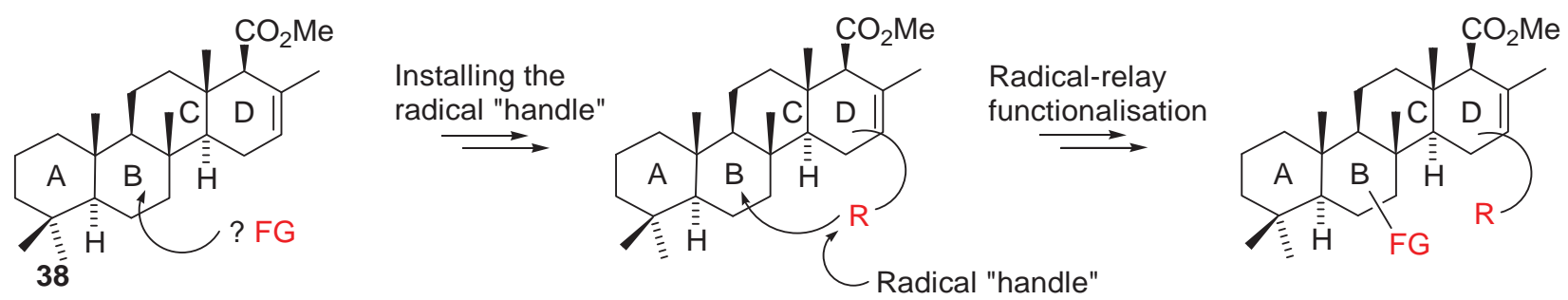

Scheme 16. Radical-relay halogenation strategy

The Scheme 17 shows implementation of the RRH for introduction of additional two functional groups in scalarane 38. According to this scheme, the starting ester was transformed into allylic alcohol 39 [24] in order to make possible an attachment of the proper radical "handle". It was introduced further on esterification with the 3-iodo-phenylacetyl chloride. The iodine atom in the aromatic cycle was necessary in order to be able to deliver specifically the chlorine radical to the definite position in the B-cycle of $\mathbf{4 0}$. The chlorine radical plays the role of the radical initiator and it efficiently substituted the tertiary proton from the B-cycle of terpenic substrate providing $4 \mathbf{4 1}$. Following elimination of hydrochloric acid generated the double bond in the B-cycle of $\mathbf{4 2}$, which can be further functionalized. In this case an allylic oxidation was performed in order to efficiently introduce the oxygen functionality in the structure $\mathbf{4 3}$ (Scheme 17). 


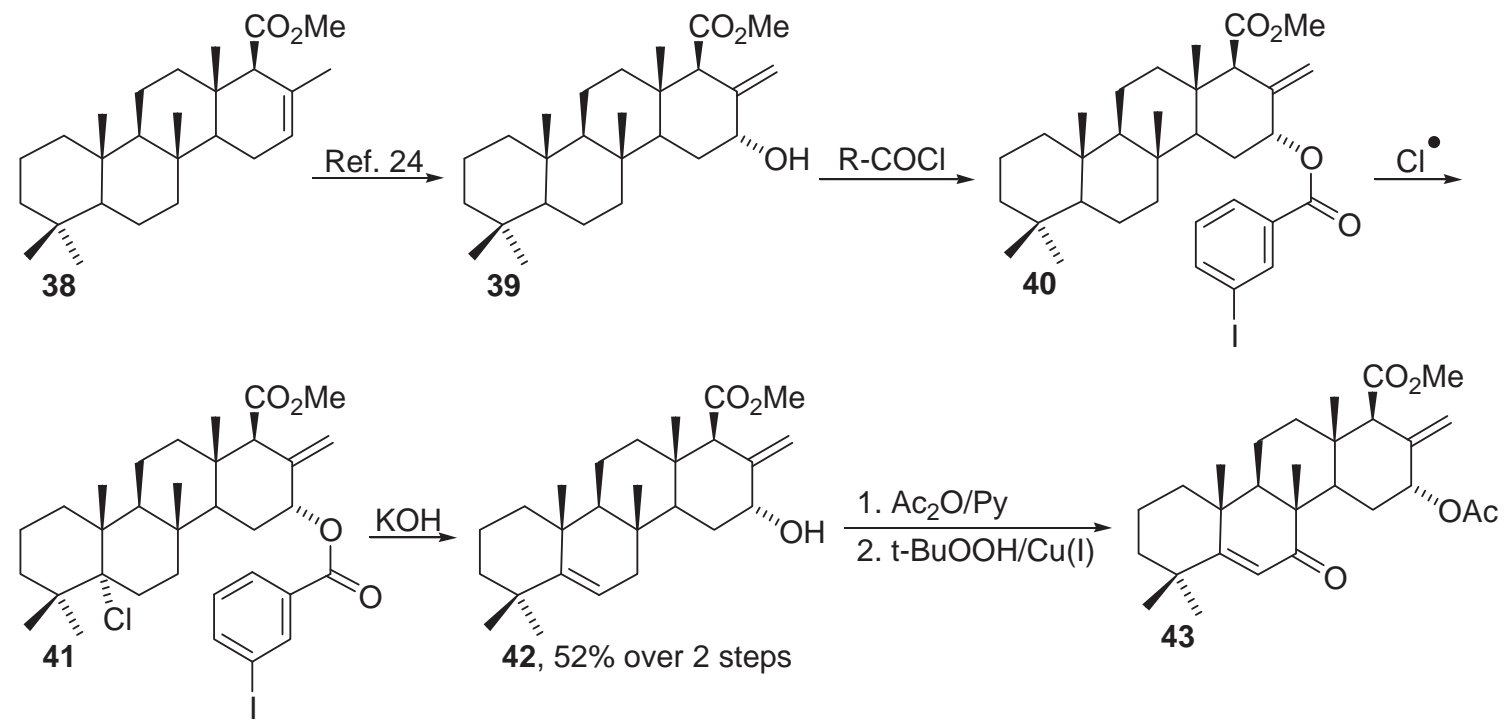

Scheme 17. Functionalisation of scalaranes by radical-relay halogenation

Finally, a pre-cyclisation terpene functionalisation was also demonstrated [4]. Functionalisation step of the substrate was achieved by a sulfone-mediated homologation of farnesylchloride 1 (Scheme 6) as discussed above. The coupling product $\mathbf{4 4}$ incorporated two heteroatom functional groups: the THP-protected hydroxyl at the $\alpha$-extremity and the phenylsulfonyl group in the middle of the molecule. Superacidic cyclisation of $\mathbf{4 4}$ led to a bicyclic compound with two pendant isoprenic units and two functional groups. As in the case of substrates with cis-configured internal double bonds described above, the phenylsulfonyl functional group contributed to suspension of cyclisation sequence, giving as the main product the sulfone $\mathbf{4 5}$.

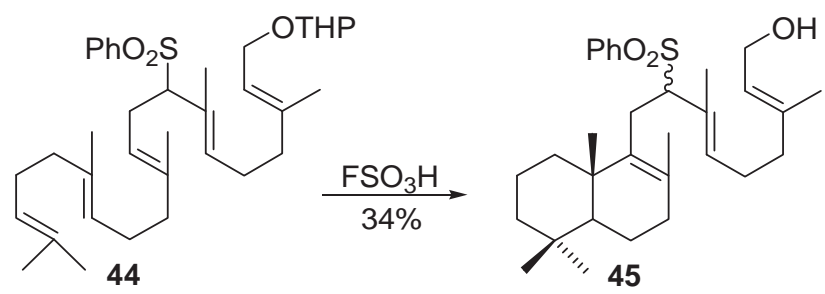

Scheme 18

\section{Conclusions}

Biomimetic strategies represent reliable tools for the elaboration of efficient synthetic pathways towards complex natural terpenes and their analogues. Most of the biosynthetical steps can be mimicked in a synthetic scheme, and different combinations of processes can lead to valuable results. Especially useful are functionalisation-cyclisationisomerisation sequences, which can assure a flexible substrate handling in a selective manner. A careful selection and placement of functional groups has allowed achieving the following selective transformations:

- Initiation of the cyclisation cascade from an internal double bond of the open chain terpenic substrate, leading to cyclic compounds with the $\omega$-prenyl group pendant;

- Suspension of the cyclisation cascade to partially cyclized compounds, having the $\alpha$-prenyl group pendant;

- Efficient functionalization of terpenic substrates either by a post-or pre-cyclisation method;

- Rearrangement of available drimanic and homodrimanic substrates to functionalized compounds of perhydrindanic structure.

Following studies are necessary in order to estimate the influence of the functionalisation pattern on the enzymatic transformations of terpene substrates. Being combined with the enzyme engineering, this approach can contribute to new biotechnological processes for selective synthesis of complex targets.

\section{References}

[1]. Topics in Current Chemistry. Biosynthesis: Aromatic Polyketides, Isoprenoids, Alkaloids. Springer Verlag, 2000, vol 209.

[2]. Prilezhaeva, E.N. Russ. Chem. Rev., 2000, 69, 367-408.

[3]. Alonso, D. A.; Najera, C. N. Desulfonylation reactions. Organic Reactions. Willey, 2009, pp. 367-656. 
[4]. Kulcițki, V.; Grinco, M.; Barba, A.; Ungur, N.; Vlad, P. Chem. Nat. Comp., 2007, 43, 268-273.

[5]. Grinco, M.; Kulcițki, V.; Ungur, N.; Barba, A.; Deleanu, C.; Vlad, P. Chem. Nat. Comp., 2007, 43, $277-281$.

[6]. Vlad, P.F. Pure Appl. Chem. 1993, 6, 1329-1336.

[7]. Kulcițki, V. Acta Biochim. Pol., 2007, 54 (4), 679-693.

[8]. Kulcițki, V.; Ungur, N.; Vlad, P.; Gavagnin, M.; Castelluccio, F.; Cimino, G. Synthesis, 2000, (3), 407-410.

[9]. Grinco, M.; Kulciţki, V.; Ungur, N.; Vlad, P.; Gavagnin, M.; Castelluccio, F.; Cimino, G. Helv. Chim. Acta, 2008, 91, 249-258.

[10]. Grinco, M.; Kulciţki, V.; Ungur, N.; Jankowski, W.; Chojnacki, T.; Vlad, P. F. Helv. Chim. Acta, 2007, 90, 12231229.

[11]. Ungur, N.; Kulcitki, V.; Gavagnin, M.; Castelluccio, F.; Vlad, P.F.; Cimino, G. Tetrahedron, 2002, 58, 1015910165.

[12]. Ungur, N.; Kulcițki, V.; Gavagnin, M.; Castelluccio, F.; Cimino, G. Synthesis, 2006, (14), 2385-2391.

[13]. Ungur, N.; Kulcițki, V. Tetrahedron, 2009, 65, 3815-3828.

[14]. Ungur, N.; Kulcițki, V. Rec. Res. Dev. Org. Chem., 2003, 7, 241-258.

[15]. Ungur, N.; Kulcițki, V. Phytochem. Rev., 2004, 3, 401-415.

[16]. Hochlowski, J.E.; Faulkner, D.J.; Matsumoto, J.; Clardy, J. J. Org. Chem., 1983, 48, 1141-1142.

[17]. Gavagnin, M.; Carbone, M.; Mollo, E.; Cimino, G. Tetrahedron Lett., 2003, 44, 1495-1498.

[18]. Kulcitki, V.; Ungur, N.; Gavagnin, M.; Carbone, M.; Cimino, G. Tetrahedron: Asymmetry, 2004, 15, $423-428$.

[19]. Kulcițki, V.; Ungur, N.; Gavagnin, M.; Carbone, M.; Cimino, G. Eur. J. Org. Chem., 2005, (9), $1816-1822$.

[20]. Kulcițki, V.; Sîrbu, T.; Ungur, N. Chem. J. Mold., 2011, 6 (1), 110-112.

[21]. Kulcitki, V.; Sirbu, T.; Barba, A.; Ungur, N. 2012, in preparation.

[22]. Kulcițki, V.; Ungur, N.; Gavagnin, M.; Castelluccio, F.; Cimino, G. Tetrahedron, 2007, 63, 7617-7623.

[23]. Breslow, R. Acc. Chem. Res., 1980, 13, 170-177.

[24]. Ungur, N.; Gavagnin, M.; Cimino, G. Nat. Prod. Lett. 1996, 8, 275-280. 